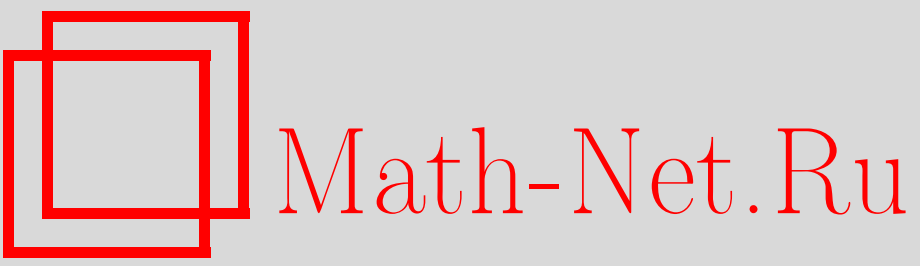

А. Н. Сесекин, А. С. Шляхов, Об одной математической модели управления инвестициями, приводящей к системе с постоянным и линейным запаздываниями, Итоги науки и техн. Сер. Соврем. мат. и ее прил. Темат. обз., 2021, том 192, 111-116

DOI: https://doi.org/10.36535/0233-6723-2021-192-111-116

Использование Общероссийского математического портала Math-Net.Ru подразумевает, что вы прочитали и согласны с пользовательским соглашением

http://www.mathnet.ru/rus/agreement

Параметры загрузки:

IP : 54.162 .85 .209

26 апреля 2023 г., 15:17:05 


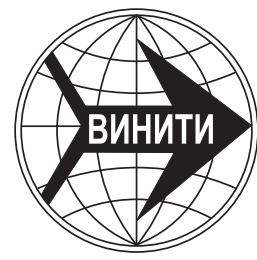

ИТОГИ НАУКИ И ТЕХНИКИ.

Современная математика и ее приложения.

Тематические обзоры.

Том 192 (2021). С. 111-116

DOI: 10.36535/0233-6723-2021-192-111-116

УДК $517.9,519.86$

\title{
ОБ ОДНОЙ МАТЕМАТИЧЕСКОЙ МОДЕЛИ \\ УПРАВЛЕНИЯ ИНВЕСТИЦИЯМИ, ПРИВОДЯЩЕЙ К СИСТЕМЕ \\ С ПОСТОЯННЫМ И ЛИНЕЙНЫМ ЗАПАЗДЫВАНИЯМИ
}

\author{
(c) 2021 г. $\quad$ А. Н. СЕСЕКИН, А. С. ШЛЯХОВ
}

\begin{abstract}
АннотАция. Предложена математическая модель управления вложениями в рекламу, которая ставит перед собой задачу учесть в себе все факторы, влияющие на процесс донесения информации. В отличие от ранее рассматривавшихся моделей, здесь рассматриваются различные способы распространения информации. Эта модель описывается дифференциальными уравнениями, которые содержат два вида запаздывания: постоянное и линейное, а также импульсное управление. Для системы такого вида формализовано понятие решения и доказана теорема существования решения.
\end{abstract}

Ключевые слова: импульсное управление, постоянное запаздывание, линейное запаздывание.

ON A MATHEMATICAL MODEL

\section{OF INVESTMENT MANAGEMENT LEADING TO A SYSTEM WITH CONSTANT AND LINEAR DELAYS}

\author{
(C) 2021 A. N. SESEKIN, A. S. SHLYAKHOV
}

\begin{abstract}
A mathematical model for managing investments in advertising is proposed, in which all the factors that affect the process of delivering information are taken into account. In contrast to earlier models, various different ways of disseminating information are discussed. The model considered is described by differential equations that contain constant delay, linear delay, and impulse control. For systems of this type, the notion of a solution is formalized and a theorem of the existence of a solution is proved.
\end{abstract}

Keywords and phrases: impulse control, constant delay, linear delay.

AMS Subject Classification: $34 \mathrm{~K} 45$

1. Управление затратами на рекламу. В основу предложенной модели легла одна из классических моделей - модель Видала-Вульфа, для которой предполагается, что изменение объемов выдач вызвано двумя составляющими: часть людей, получивших информацию о продукте через рекламу, забывает ее, другие покидают рынок по каким-либо иным причинам с постоянным темпом. Увеличение продаж продукта пропорционально вложениям в рекламу, а также части рынка, которая не охвачена фирмой, либо принадлежит конкурирующей компании. В общем случае модель описывается следующим уравнением:

$$
\dot{S}(t)=k \cdot u(t) \cdot\left(1-\frac{S(t)}{M}\right)-b \cdot S(t), \quad S(0)=S_{0}
$$


где $S(t)$ - текущий объем продаж, $u(t)$ - текущий поток инвестиций в рекламу, $b$ - темп, с которым уменьшается объем продаж $(b=\mathrm{const}), M$ - максимально возможный объем продаж (включая конкурентов, людей, не обладающих достаточным уровнем информации и т. д., $M=\mathrm{const})$, $k$ - темп, с которым прирастает объем выдач $(k=\mathrm{const})$.

Реализация инновационных товаров обладает определенными особенностями. Первое время потребитель не знает о товарах и его преимуществах перед клиентом, поэтому производителю приходится проводить активную рекламную акцию. Кроме того, покупатели, узнав о товаре, не сразу его покупают, в силу индивидуальных особенностей.

В 1943 году Райн и Гросс (Ryan и Gross) в ходе исследовании процесса распространения новых сортов сельскохозяйственных культур среди фермеров предложили следующую классификацию потребителей в зависимости от скорости принятия решения о приобретении нового товара на рынке (см. [8]):

1. Новаторы $(2,5 \%)$ - люди, которые наиболее подготовлены к изменениям и покупают товар сразу же, без раздумий.

2. Ранние последователи $(13,5 \%)$ - люди, которые приобрести новый товар рано, но при этом соблюдая некоторую осторожность.

3. Раннее большинство $(34 \%)$ - потребители, которые покупают раньше среднего, но при этом они максимально приближены к среднему значению.

4. Позднее большинство (34\%) - люди, которые настроены скептически к новым идеям, но все же со временем принимают их.

5. Отстающие $(16 \%)$ - консерваторы, воспринимающие идеи с трудом и недоверием.

В работе В. В. Цыганова и Ю. Г. Бочкаревой [4] описывается модель распространения информации. В работе подчеркивается важность комплексного рассмотрения распространения информации, которая учитывает различные каналы распространения информации, в том числе канал «из уст в уста». Распространение информации по этому каналу происходит с запаздыванием, поскольку со временем запаздывание изменяется линейно, ввиду уменьшения интереса потребителей к товару.

Во многих работах описывается влияние на продажи человеческого фактора, но все модели предполагают, что после получения информации люди сразу же идут приобретать товар. На практике же между получением информации и покупкой товара проходит некоторое время, которое и определяет 5 основных групп потребителей. Это время включает в себя множество составляющих, таких как, например:

(i) сомнения в качестве товара;

(ii) время на обсуждение покупки с близкими;

(iii) время на сбор средств для покупки товара;

(iv) время на оформление товара.

В конечном итоге, можно условно разделить все факторы на 2 группы:

(а) факторы, в которых временные затраты постоянные (т.е. при реализации товара возникает постоянное запаздывание) (например, (iii) и (iv));

(b) факторы, в которых временные затраты изменяются линейно со временем (т.е. при реализации товара возникает линейное запаздывание) (например, (i) и (ii)).

Остановимся подробнее на факторах с линейным запаздыванием. Эти факторы зависят от индивидуальных особенностей каждого человека, ведь в зависимости от категории людей, время, которое тратится на принятие решения, разнится. При этом, чем больше времени прошло с начала рекламной кампании, тем больше времени тратит оставшаяся категория людей на принятие решения. Модель Видала-Вульфа показывает достаточно хорошие результаты, но не учитывает реакцию потребителей на полученную информацию о товаре. В этой связи предлагается усложнить данную модель, вводя новую переменную, определяющую распространение информации и, как следствие, количество потенциальных покупателей. Такое усложнение модели позволит связать ее с идеями Райана и Гросса, а также учесть распространение информации по каналу «из уст в уста». Пусть $S(t)$ - количество людей, которые становятся потенциальными покупателями в момент времени $t, P(t)$ - количество продаж продукта в момент времени $t$. 
Предлагается следующая модель:

$$
\left\{\begin{array}{l}
\dot{S}(t)=k_{1} \cdot u(t) \cdot\left(1-\frac{S(t)}{M}\right)-b_{1} \cdot S(t)+d \cdot P(t), \quad S\left(t_{0}\right)=S_{0}, \\
\dot{P}(t)=k_{2} \cdot S(t)+k_{3} \cdot S(t-\tau)+k_{4} \cdot S(\mu \cdot t)-b_{2} \cdot P(t),
\end{array}\right.
$$

где $u(t)$ - текущий объем инвестиций в рекламу, $b_{1}, b_{2}$ - темпы, с которым уменьшаются потенциальное количество потребителей и объем продаж, соответственно $\left(b_{i}=\mathrm{const}\right), M-$ максимально возможное количество человек в целевой аудитории ( $M=\mathrm{const}), k_{1}$ - темп, с которым прирастает количество потенциальных покупателей, которые узнали о продукте из рекламы $\left(k_{1}=\mathrm{const}\right)$, $d$ - темп, с которым люди, приобретшие товар, возвращаются повторно, $k_{2}$ - темп, с которым потенциальные покупатели, которые относятся к категории «новаторы», приобретают товар, $k_{3}-$ темп, с которым покупают товар люди из категории «ранние последователи», $k_{4}-$ темп, с которым товар приобретают оставшиеся категории потребителей, $\tau$ - среднее время запаздывания, с которым «ранние последователи» приходят за товаром, после того как узнают о нем, $\mu-$ коэффициент замедления отклика потенциальных покупателей со временем.

Компании могут вкладывать средства непрерывно, но зачастую вложения происходят более дискретно. В последнем случае на систему будет действовать импульсное управление (см. [2]), а траектория становится разрывной. Аналогичные исследования в части импульсного управления приводились для задачи управления рекламными расходами в случае продажи двух взаимодополняемых продуктов (см. [1]). Перечисленные выше модели см. в $[2,4,6,8]$.

2. Формализация и существование решений для системы с двумя запаздываниями. Полученная ранее модель принадлежит следующему классу уравнений:

$$
\dot{x}(t)=f(t, x(t), x(t-\tau), x(\mu t))+b(t, x(t)) \dot{v}(t), \quad t \in\left[t_{0}, \vartheta\right] .
$$

Начальное условие будем задавать следующим образом:

$$
x(t)=\varphi(t), \quad t \in\left[\min \left\{t_{0}-\tau, \mu t\right\} ; t_{0}\right], \quad t_{0} \geqslant 0, \quad \tau>0, \quad 0<\mu<1 .
$$

Здесь $x(t)$ - $n$-вектор-функция, $v(t)$ - скалярная функция времени, $f(t, x, y, z)$ и $b(t, x)-n$-вектор-функции, $v(\cdot) \in B V\left[t_{0}, \vartheta\right]$, где $B V\left[t_{0}, \vartheta\right]$ - банахово пространство скалярных вектор-функций ограниченной вариации. Предположим, что $f(\cdot, \cdot, \cdot, \cdot)$ измерима по $t$ и липшицева по $x, y, z$, т.е.

$$
\|f(t, x, y, z)-f(t, \bar{x}, \bar{y}, \bar{z})\| \leqslant L(\|x-\bar{x}\|+\|y-\bar{y}\|+\|z-\bar{x}\|),
$$

а $b(\cdot, \cdot)$ непрерывна по совокупности переменных и липшицева по $x$, т.е.

$$
\|b(t, x)-b(t, \bar{x})\| \leqslant L(\|x-\bar{x}\|)
$$

на множестве $\left\{t \in\left[t_{0}, \vartheta\right],\|x\|<\infty,\|y\|<\infty,\|z\|<\infty,\|v\|<\infty\right\}$, где

$$
\|x\|=\left(\sum_{i=1}^{n}\left|x_{i}\right|^{2}\right)^{1 / 2},
$$

и удовлетворяют следующим условиям:

$$
\|f(t, x, y, z)\| \leqslant \kappa(1+\|x\|+\|y\|+\|z\|), \quad\|b(t, x)\| \leqslant \kappa(1+\|x\|),
$$

где $\kappa$ - некоторая положительная постоянная.

Далее нам понадобится интегральная форма уравнения (3)

$$
x(t)=\varphi\left(t_{0}\right)+\int_{t_{0}}^{t} f(s, x(s), x(s-\tau), x(\mu s)) d s+\int_{t_{0}}^{t} b(s, x(s)) d v(s), \quad t \in\left[t_{0}, \vartheta\right] .
$$

Особенностью уравнения (3) является то, что в правой части уравнения присутствует некорректная операция умножения разрывной функции на обобщенную. Дело в том, что если функция $v(t)$ будет разрывной, то ее обобщенная производная будет содержать дельта-функцию, а наличие ее в правой части уравнения (3) приведет к разрывности решения $x(t)$. Отмеченная проблема, как и в $[3,7,9]$, решается с помощью процедуры замыкания множества гладких решений в пространстве функций ограниченной вариации. 
Пусть $v_{k}(t) \in A C_{m}\left[t_{0}, \vartheta\right], k=1,2, \ldots$, где $A C_{m}\left[t_{0}, \vartheta\right]$ - банахово пространство абсолютно непрерывных функций. $v_{k}(t)$ поточечно сходится к $v(t) \in B V_{m}\left[t_{0}, \vartheta\right], \forall t \in\left[t_{0}, \vartheta\right]$. При сделанных предположениях решение задачи Коши (3), (4) согласно [5, с. 31] существует и единственно для $\forall v_{k}(t) \in A C\left[t_{0}, \vartheta\right],\left(v(t)\right.$ и $v_{k}(t)(k=1,2, \ldots)$. Будем выбирать последовательности $v_{k}(t)$ из множества функций, удовлетворяющих ограничению $\left.\underset{\left[t_{0}, \vartheta\right]}{\operatorname{var}} v(\cdot) \leqslant a\right)$. Обозначим через $x_{k}(t)$ решения задачи Коши (3), (4), порожденные последовательностью $v_{k}(t), k=1,2, \ldots$

Определение 1. Как и в [9], вектор-функцию $x(t) \in B V_{n}\left[t_{0}, \vartheta\right]$ будем называть аппроксимируемым решением задачи Коши (3), (4), если $x(t)$ - поточечный предел последовательности $x_{k}(t)$, $k=1,2, \ldots$, абсолютно непрерывных функций, порожденной последовательностью абсолютно непрерывных функций $v_{k}(t)$, поточечно сходящейся к функции $v(t)$, и $x(t)$ не зависит от выбора последовательности $v_{k}(t)$.

Теорема 1. При сделанных выше предположения $\forall v(t) \in B V\left[t_{0}, \vartheta\right]$ существует аппроксимируемое решение $x(t)$ уравнения (3), которое удовлетворяет интегральному уравнению

$$
\begin{aligned}
x(t)=x\left(t_{0}\right)+\int_{t_{0}}^{t} f(\xi, & x(\xi), x(\xi-\tau), x(\mu \xi)) d \xi+\int_{t_{0}}^{t} B(\xi, x(\xi)) d v^{c}(\xi)+ \\
& +\sum_{t_{i} \leqslant t, t_{i} \in \Omega_{-}} S\left(t_{i}, x\left(t_{i}-0\right), \Delta v\left(t_{i}-0\right)\right)+\sum_{t_{i}<t, t_{i} \in \Omega_{+}} S\left(t_{i}, x\left(t_{i}\right) \Delta v\left(t_{i}+0\right)\right),
\end{aligned}
$$

где $v^{c}(\xi)$ - непрерывная составляющая функции $v(\xi), \Omega_{-}\left(\Omega_{+}\right)$- множества точек, в которых функция v(t) разрывна слева (справа),

$$
S(t, x, \Delta v)=z(1)-z(0), \quad \dot{z}(\xi)=B(t, x(\xi)) \Delta v(t), \quad z(0)=x .
$$

Доказательство. Вычислим нормы левой и правой частей уравнения (8) и воспользуемся оценками (7). В результате получим

$$
\left\|x_{k}(t)\right\| \leqslant\left\|\varphi\left(t_{0}\right)\right\|+\int_{t_{0}}^{t} \kappa(1+\|x(s)\|+\|x(s-\tau)\|+\|x(\mu s)\|) d s+\int_{t_{0}}^{t} \kappa(1+\|x(s)\|) d \underset{\left[t_{0}, s\right]}{\operatorname{var}} v_{k}(\cdot) .
$$

Из последней оценки очевидным образом имеем

$$
\left\|x_{k}(t)\right\| \leqslant\left\|\varphi\left(t_{0}\right)\right\|+\int_{t_{0}}^{t} \kappa(1+2\|x(s)\|+\|x(s-\tau)\|+\|x(\mu s)\|) d\left(s+\operatorname{var}_{\left[t_{0}, s\right]} v_{k}(\cdot)\right) .
$$

Введем обозначение

$$
h_{k}(t)=\sup _{[\min \{t-\tau, \mu t\}, t]}\left\|x_{k}(\cdot)\right\| .
$$

Тогда из (10) с учетом (11) следует

$$
h_{k}(t) \leqslant h_{k}\left(t_{0}\right)+\int_{t_{0}}^{t} \kappa\left(1+4 h_{k}(s)\right) d\left(s+\operatorname{var}_{\left[t_{0}, s\right]} v_{k}(\cdot)\right) .
$$

Это неравенство можно переписать в виде

$$
h_{k}(t) \leqslant h_{k}\left(t_{0}\right)+\kappa\left(t-t_{0}+\operatorname{var}_{\left[t_{0}, t\right]} v_{k}(\cdot)\right)+4 \kappa \int_{t_{0}}^{t} h_{k}(s) d\left(s+\operatorname{var}_{\left[t_{0}, s\right]} v_{k}(\cdot)\right) .
$$


Применяя к этому неравенству лемму 5.4.3 из [9], получим оценку

$$
\begin{aligned}
h_{k}(t) \leqslant h_{k}\left(t_{0}\right)+ & 4 \kappa \exp \left(\kappa\left(t+\underset{\left[t_{0}, t\right]}{\operatorname{var}} v_{k}(\cdot)\right)\right) \times \\
& \times \int_{t_{0}}^{t}\left[h_{k}(s)+\kappa\left(s-t_{0}+\underset{\left[t_{0}, s\right]}{\operatorname{var}} v_{k}(\cdot)\right)\right] \exp \left(-\kappa\left(s+\operatorname{var}_{\left[t_{0}, s\right]}^{\operatorname{var}} v_{k}(\cdot)\right)\right) d\left(s+\operatorname{var}_{\left[t_{0}, s\right]} v_{k}(\cdot)\right) .
\end{aligned}
$$

В силу условий $t \in\left[t_{0}, \vartheta\right], \underset{\left[t_{0}, t\right]}{\operatorname{var}} v_{k}(\cdot) \leqslant a$ из $(12)$ получаем

$$
h_{k}(t) \leqslant\left(h_{k}\left(t_{0}\right)+4 \kappa\left(\vartheta-t_{0}+a\right)\right) e^{\kappa\left(\vartheta-t_{0}+a\right)}=c_{1} .
$$

Отсюда следует равномерная ограниченность $h_{k}(t)$, а в силу (11) и равномерная ограниченность $x_{k}(t)$ на $\left[t_{0}, \vartheta\right]$, т.е.

Согласно (3)

$$
x_{k}(t) \leqslant c_{1} .
$$

$$
\operatorname{var}_{\left[t_{0}, t\right]} x_{k}(\cdot)=\int_{t_{0}}^{t}\left\|x_{k}(s)\right\| d s \leqslant \int_{t_{0}}^{t}\|f(s, x(s), x(s-\tau), x(\mu s))\| d s+\int_{t_{0}}^{t}\|b(s, x(s))\| d \underset{\left[t_{0}, s\right]}{\operatorname{var}} v_{k}(\cdot) .
$$

Из $(7)$ и ограниченности $x_{k}(t)$ на $\left[t_{0}, \vartheta\right]$ из (15) следует равномерная ограниченность $\operatorname{var} x_{k}(\cdot)$.

Последовательность абсолютно непрерывных функций $v_{k}(t)$ на $\left[t_{0}, \vartheta\right]$ поточечно сходится в функции ограниченной вариации $v(t)$. Порожденная последовательностью $v_{k}(t)$ последовательность абсолютно непрерывных функций $x_{k}(t)$, как показано выше, равномерно ограничена (14) и равномерно ограничена последовательность их вариаций (15). Поэтому согласно теореме Хелли (см. [9]) из этой последовательности можно выделить сходящуюся поточечно к некоторой функции ограниченной вариации $x(t)$ подпоследовательность $x_{k_{i}}(t)$.

Функции $v_{k_{i}}(t)$ и $x_{k_{i}}(t)$ удовлетворяют уравнению

$$
x_{k_{i}}(t)=\varphi\left(t_{0}\right)+\int_{t_{0}}^{t} f\left(s, x_{k_{i}}(s), x_{k_{i}}(s-\tau), x_{k_{i}}(\mu s)\right) d s+\int_{t_{0}}^{t} b\left(s, x_{k_{i}}(s)\right) d v_{k_{i}}(s), \quad t \in\left[t_{0}, \vartheta\right] .
$$

Перейдем в уравнении (16) к пределу при $k_{i} \rightarrow \infty$. Переход к пределу в первом интеграле в (16) в согласно теореме Лебега о предельном переходе под знаком интеграла очевиден. Во втором интеграле предельный переход можно осуществить аналогично $[3,7,9]$, используя замену времени $\xi=t+\operatorname{var} v_{k}(\cdot)$. Эта возможность обеспечивается тем, что $b(t, x(t))$ не зависит от $x(t-\tau)$ и $x(\mu t)$. В результате убеждаемся, что $x(t)$ и $v(t)$ удовлетворяют уравнению (8).

Покажем, что решение уравнения (8) единственно. Предположим противное, существуют два решения уравнения $(8) x(t)$ и $\bar{x}(t)$. Используя $(5),(6)$ и $(7)$ оценки на величину скачков траектории из [9], получаем следующее неравенство:

$$
\begin{aligned}
\|x(t)-\bar{x}(t)\| \leqslant \int_{t_{0}}^{t} L(\|x(s)-\bar{x}(s)\|+\|x(s-\tau)-\bar{x}(s-\tau)\|+\|x(\mu s)-\bar{x}(\mu s)\|) d s+ \\
+\int_{t_{0}}^{t} L(\|x(s)-\bar{x}(s)\|) d \operatorname{var}_{\left[t_{0}, s\right]} v(\cdot)+\sum_{t_{i} \leqslant t, t_{i} \in W_{-}}\left(e^{L\left|\Delta v\left(t_{i}-0\right)\right|}-1\right)\left\|x\left(t_{i}-0\right)-\bar{x}\left(t_{i}-0\right)\right\|+ \\
+\sum_{t_{i}<t, t_{i} \in W_{+}}\left(e^{L\left|\Delta v\left(t_{i}+0\right)\right|}-1\right)\left\|x\left(t_{i}\right)-\bar{x}\left(t_{i}\right)\right\| .
\end{aligned}
$$

Введем обозначение

$$
g(t)=\sup _{[\min \{t-\tau, \mu t\}, t]}\|x(\cdot)-\bar{x}(\cdot)\| .
$$


Используя (18), мы можем из (17) получить неравенство

$$
\begin{aligned}
g(t) \leqslant \int_{t_{0}}^{t} 3 L g(s) d(s & \left.+\operatorname{var}_{\left[t_{0}, s\right]} v(\cdot)\right)+ \\
& +\sum_{t_{i} \leqslant t, t_{i} \in W_{-}}\left(e^{L\left|\Delta v\left(t_{i}-0\right)\right|}-1\right) g\left(t_{i}-0\right)+\sum_{t_{i}<t, t_{i} \in W_{+}}\left(e^{L\left|\Delta v\left(t_{i}+0\right)\right|}-1\right) g\left(t_{i}\right) .
\end{aligned}
$$

Применяя к (19) лемму 5.4.3 из [9], получаем неравенство $g(t) \leqslant 0$, что и завершает доказательство единственности решения уравнения (8). Из факта единственности решения уравнения (8) следует независимость решения $x(t)$ от способа аппроксимации функции $v(t)$.

3. Заключение. Оптимальное управление затратами на рекламу при реализации новых продуктовых линеек является одной из важнейших задач для предприятий. Недостаточные вложения в рекламу могут привести к тому, что потенциальные потребители инновационного товара не получат информацию о нем, и компания потеряет клиента. С другой стороны, чрезмерные вливания денежных средств в рекламную кампанию могут принести существенные убытки. Кроме того, при определении оптимальных инвестиций в распространение информации о продукте следует учитывать конкуренцию на рынке. В работе предлагается модель управления вложениями в рекламу, которая ставит перед собой задачу учесть в себе все факторы, влияющие на процесс донесения информации, а именно, процесс принятия решения разными группами потребителей, конкуренцию на рынке, диффузию инноваций. Отличительной особенностью предложенной математической модели является то, что дифференциальные уравнения, описывающие эту модель, содержат два вида запаздывания: постоянное и линейное, а также импульсное управление. Рассмотрена система дифференциальных уравнений, частным случаем которой является предложенная модель. Для этой системы формализовано понятие решения и доказана теорема существования и единственности решения.

\section{СПИСОК ЛИТЕРАТУРЫ}

1. Баенхаева В. А. Исследование оптимального импульсного управления в модели рекламных расходов// Вестн. Бурят. гос. ун-та. Мат. Информ. - 2009. - 9. - С. 18-21.

2. Дыхта В. А., Самсонюк О. Н. Оптимальное импульсное управление с приложениями. - М.: Физматлит, 2003.

3. Сесекин А. Н. Динамические системы с нелинейной импульсной структурой// Тр. ин-та мат. мех. УрО PAH. - 2000. - 6, № 2. - C. 497-514.

4. Цыганов В. В., Бочкарева Ю. Г. Комплексный подход к методологии распространения информации// Фундам. исслед. - 2013. - 4, № 3. - С. 612-617.

5. Эльсгольи, Л. Э., Норкин С. Б. Введение в теорию дифференциальных уравнений с отклоняющимся аргументом. - М.: Наука, 1971.

6. Dorroh J. R., Ferreyra G. A multistate, multicontrol problem with unbounded controls// SIAM J. Control Optim. - 1994. - 32, № 5. - P. 1322-1331.

7. Miller B. M., Rubinovich E. Ya. Discontinuous solutions in the optimal control problems and their representation by singular space-time transformations// Automat. Remote Control. — 2013. - 74. - P. 1969-2006.

8. Ryan B., Gross N. The diffusion of hybrid seed corn in two Iowa communities// Rural Sociology. - 1943. - 8 (1). - P. $15-24$.

9. Zavalishchin S. T., Sesekin A. N. Dynamic Impulse Systems: Theory and Applications. — Dordrecht: Kluwer Academic, 1997.

Сесекин Александр Николаевич

Уральский федеральный университет

им. первого Президента России Б. Н. Ельцина, Екатеринбург;

Институт математики и механики им. Н. Н. Красовского УрО РАН, Екатеринбург

E-mail: sesekin@list.ru

Шляхов Александр Сергеевич

Публичное акционерное общество «Сбербанк России»

E-mail: aleksandr.shlyakhov@gmail.com 